\title{
Investigation of short-side-chain ionomer and membrane for proton exchange membrane fuel cells
}

\author{
Samaneh Shahgaldi \\ Department of Mechanical and Mechatronics Engineering, \\ University of Waterloo, 200 University Avenue West, \\ Waterloo, ON N2L 3G1, Canada \\ samaneh.shahgaldi@uwaterloo.ca
}

\author{
Adnan Ozden \\ Department of Mechanical and Mechatronics Engineering, \\ University of Waterloo, 200 University Avenue West, \\ Waterloo, ON N2L 3G1, Canada \\ aozden@uwaterloo.ca
}

\author{
Xianguo Li \\ Department of Mechanical and Mechatronics Engineering, University of Waterloo, \\ 200 University Avenue West, Waterloo, ON N2L 3G1, Canada \\ xianguo.li@uwaterloo.ca
}

\begin{abstract}
Proton exchange membrane (PEM) fuel cells have been progressively designed to become suitable for hightemperature operation to achieve further performance improvements. However, the current state-of-the-art fuel cell materials, such as long-side-chain (LSC) ionomers and membranes, are not suitable for high-temperature operation, requiring development and investigation of alternative materials. In this study, short-side-chain (SSC) membrane and ionomer are considered as potential materials, and performance of a membrane-electrode assembly (MEA) manufactured with the SSC ionomer and membrane is experimentally investigated in a scaled-up fuel cell $\left(45 \mathrm{~cm}^{2}\right)$. Comparison is made with an MEA based on the LSC ionomer and membrane under identical preparation and testing conditions. The catalyst layers (CLs) made of either SSC or LSC ionomer are characterized through scanning electron microscopy (SEM) to understand their surface morphology and microstructure. Results show that the SSC ionomer embedded in the CL provides much more uniform surface morphology and well-proportioned microstructural characteristics than its LSC counterpart. Further, the MEA based on SSC ionomer and membrane demonstrates considerable performance superiorities under all the applied operating conditions. Furthermore, the performance of the MEA based on the SSC ionomer and membrane is found to be less sensitive to changes in operating conditions.
\end{abstract}

Keywords- Proton exchange membrane fuel cell; Short-sidechain ionomer; Short-side-chain membrane; High-temperature fuel cell operation

\section{INTRODUCTION}

Proton exchange membrane (PEM) fuel cells are an energy-conversion device that converts the chemical energy of hydrogen and oxygen into electricity through electrochemical reactions. In recent years, PEM fuel cell technology has perceivably advanced and reached the early stage of commercialization, but along with remaining technical challenges to be overcome for further improvements in performance [1-3]. To overcome many of these challenges, fuel cells have been gradually designed to provide trouble-free performance at high operating temperatures.

Fuel cells adopted for low-temperature operation are highly susceptible to carbon monoxide (CO) poisoning, since the adsorption of $\mathrm{CO}$ on the catalyst nanoparticles is favored at low temperatures [4]. Thus, high-temperature operation would certainly increase the $\mathrm{CO}$ tolerance, which can in turn eliminate prohibitive technological costs through catalyst loading reduction. Further, a fuel cell generates a considerable amount of heat due to electrochemical reactions and associated irreversibilities that needs to be effectively removed to maintain the operating temperature, requiring the development of effective cooling strategies. Thus, if the cell is adopted for high-temperature operation, there will be no necessity for well-planned cooling strategies. Besides, the overall system efficiency can be substantially boosted through recovering of excess heat as steam for direct heating or pressurized conditions. High-temperature operation can also substantially minimize the damage caused by fuel impurities [5], as it does not require high-level of humidification for pressurization, unlike low-temperature operation, and hence the problems associated with water management can also be mitigated. High-temperature operation can also simultaneously increase the diffusion rate of the reactants and simplify detachment of water molecules from the catalyst layer (CL) - which would consequently facilitate the diffusion of the reactants into the three-phase boundary.

Although high-temperature operation provides the aforementioned promises, today's fuel cells perform only well at low temperatures, typically $60-80^{\circ} \mathrm{C}$ [6]. This restriction originates from unsuitableness of the archetypal membranes and ionomers for high-temperature operation (i.e., long-side chain (LSC) membranes and ionomers), since they are 
typically made of perfluorosulfonic acid. Dupont's Nafion ${ }^{\circledR}$ can be given as a good example of these ionomers and membranes, in which proton transport is governed by "Vehicular" and "Grotthus" mechanisms [7,8] - the mechanisms function only well in the presence of adequate water, unlikely to be seen at high temperatures [9]. Recently, several alternatives, known as short-side chain (SSC) ionomers and membranes, have been introduced into the fuel cell market to make fuel cells viable for high-temperature operation (see [10], for example). The SSC ionomers and membranes can provide favorable thermal transition temperature and ion exchange capacity (EIC), without facing any swelling and/or dissolution [11]. These characteristics also enable them to absorb and retain water even at elevated temperatures. Even though the SSC ionomers and membranes are popular owing to their suitability for high-temperature operation, their cell performance characteristics are still under vehement investigations.

The objective of the present work is therefore to investigate cell performances of the SSC ionomers and membranes under different cell conditions: varied cell temperatures, varied anode and cathode flow rates, and varied anode and cathode relative humidities (RHs). To understand the morphological and microstructural differences between the SSC and LSC ionomers, scanning electron microscopy (SEM) analyses of the CLs made of these ionomers are performed. For comparison, cell performances of the membrane-electrode assemblies (MEAs) with the SSC ionomer-based CLs and membranes are evaluated. Cell performances are compared with those of the MEAs based on the LSC-based ionomers and membranes under the same conditions.

\section{EXPERIMENTAL}

The catalyst inks are prepared by using carbon-supported platinum (50\% Pt to $\mathrm{C}$ ratio, Tanaka Kikinzoku Kogyo K.K.) as a catalyst, either short-side-chain ionomer (Aquivion ${ }^{\circledR}$ with equivalent weight of $790 \mathrm{~g} \mathrm{eq}^{-1}$ ) or long side chain ionomer ( Nafion (EW) $1100 \mathrm{~g} \mathrm{eq}^{-1}$ ) as a binding agent, and 2-proponal (IPA, 99.9\%, Sigma ${ }^{\circledR}$-Aldrich) as a solvent. Proper amount of these materials is mixed in a $20 \mathrm{ml}$ vial, followed by mechanical mixing in an ultrasonic bath for $1 \mathrm{~h}$. The PtC/ionomer ratio in the electrodes is kept constant as 3.0/1.0. The ink prepared is deposited onto the selected membrane (either Aquivion E87-05S with equivalent weight of $890 \mathrm{~g} \mathrm{eq}^{-1}$ or Nafion 211) via catalyst-coated membrane (CCM) method. The CCM is then sandwiched between the anode and cathode gas diffusion layers (GDLs, Avcarb GDS 3250) to obtain the MEA. The electrode active areas of the MEAs are $45 \mathrm{~cm}^{2}$, and the Pt loadings in the anode and cathode electrodes are kept constant as $0.10 \mathrm{mg} / \mathrm{cm}^{2}$ and $0.40 \mathrm{mg} / \mathrm{cm}^{2}$, respectively. Cell performances of the MEAs based on different ionomers and membranes are obtained under the same conditions: a varied cell temperature, varied anode and cathode flow rates, and varied anode and cathode relative humidities. The polarization curves are obtained by potentiostatically reducing the cell voltage and recording the corresponding current values. Each polarization curve is the average of three polarization curves obtained on three different days, with a standard deviation no more than $5 \%$.

\section{RESULTS AND DISCUSSION}

\section{A. Morphological and Microstructural Characteristics of the Catalyst Layers}

Figs. 1 (a) and (b) present the top view of the CLs made of the SSC and LSC ionomers. Both the CLs have similar compact and porous surface characteristics - such characteristics are quite common for the CLs made of Pt-C catalyst. Since the CLs are subjected to gold coating prior to SEM analyses, the cracks apparent on the surface could be associated with the gold deposition. Darker particles in the images show the Pt-C particles, while black holes represent the pores existing in the regions near the surface. Ionomer surrounding the $\mathrm{Pt}-\mathrm{C}$ particles is shown by lighter contrast in the images. Clearly, both the CLs have homogeneous surface characteristics, but the SSC ionomer-based CL seems to have relatively more open pores, along with virtually no ionomer agglomeration (see Fig. 1(a)), clearly indicating the SSC ionomer's compatibility with the Pt-C particles. Such compatibility could be related to inherent binding characteristics of the SSC ionomer. In contrast, in the LSC ionomer-based CL, the ionomer films are more prone to accumulate and build up bigger ionomer-ionomer agglomerates (see Fig. 1(b)), which is not so desirable, because such agglomerates shrink the available pores that are available for mass transport. Therefore, compared to the LSC ionomer-based CL, the SSC ionomer-based one has relatively more open pores. The pore characteristics of the electrodes based on these CLs are investigated by the Method of Standard Porosimetry (MSP) under identical testing conditions, and results are in good agreement with the SEM images. It is seen that the SSC ionomer-based CL has relatively a relatively higher porosity $(73.10 \%)$ than the LSC ionomer-based one $(68.80 \%)$.

\section{B. Fuel Cell Performance Testing}

Scaled-up single cell performance tests are conducted to provide practical insights into the differences between the CLs and membranes made of the SSC and LSC ionomers, under various operating conditions, i.e., different cell temperatures and different flow rates, and relative humidities (RHs) of air and hydrogen. For the virtue of repeatability, each polarization curve is obtained from measurements conducted on three different days under identical conditions. Figs. 2 (a) and (c) compare the polarization and power density curves of the MEAs with the CLs made of two different ionomers - SSC and LSC - as polymeric binders and membranes as a function of varying air and hydrogen flow rates. The other testing parameters are held constant, such that the cell temperature is set as $75^{\circ} \mathrm{C}$, while the back pressures and RHs of the hydrogen and air streams are set as $35 \mathrm{kPag}$ and $100 \%$, respectively. Two different flow rates of hydrogen and air are applied. Fixed anode and cathode flow rates of 4.45 and $9.00 \mathrm{l} / \mathrm{min}$ applied is named as "high flow", while the anode and cathode stoichiometries of $1.20 / 2.00$ is named as "low flow", throughout the rest of this paper. As evident from Fig. 2 (a), the MEA with the SSC-ionomer based CLs and membrane is relatively less sensitive to flow rates of the reactants. The open circuit voltages (OCVs) obtained from this MEA at the highand low-flow rates are almost the same, while there is a slight 
difference between the current densities when the cell voltage is decreased, such that the maximum power densities obtained under high- and low-flow conditions are $0.96 \mathrm{~W} / \mathrm{cm}^{2}$ and 0.94 $\mathrm{W} / \mathrm{cm}^{2}$, respectively. On the other hand, the MEA with the LSC-based CLs and membrane shows almost the same performance at low current densities under both the high- and low-flow conditions, as seen in Fig. 2 (b). However, a noticeable reduction in cell performance is observed, as the cell is operated at high current densities, and the reactants are supplied under low-flow conditions. This trend indicates that the LSC-based CL and membrane provide conditiondependent performance, further suggesting that the LSC-based components are highly sensitive to changes in operating conditions. This sensitivity probably originates from the dimensional changes in the LSC ionomer; the LSC ionomer potentially experiences severe swelling at high current densities, blocking the pores available for the mass transport. In contrast, the MEA with the SSC ionomer-based CLs and membrane shows almost the same performance, regardless of flow conditions, indicating the stability of this ionomer, which potentially experiences less swelling, as the current density is increased. Such behaviour probably preserves the porosity of the electrode, thus almost the same mass transport capability is

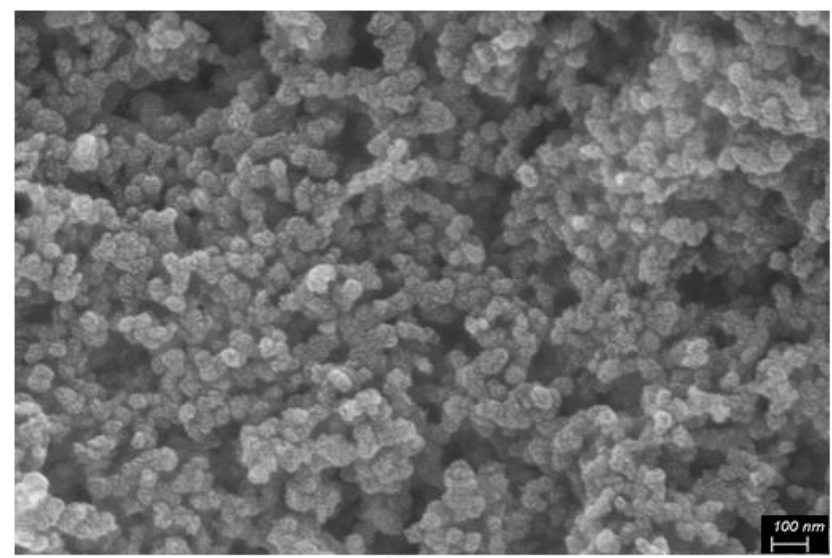

(a)

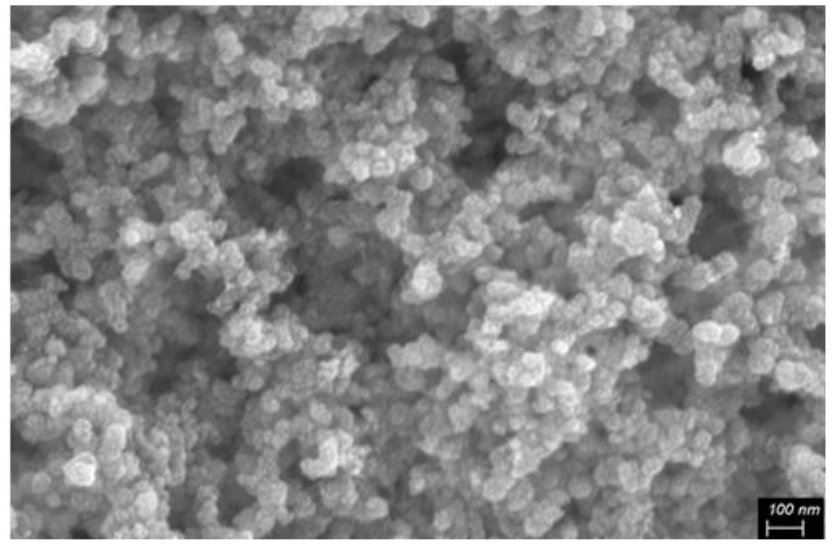

(b)

Figure 1. Scanning electron microscopy (SEM) images of the catalyst layers (CLs) made of (a) short-side chain (SSC) ionomer and (b) long-side-chain (LSC) ionomer.
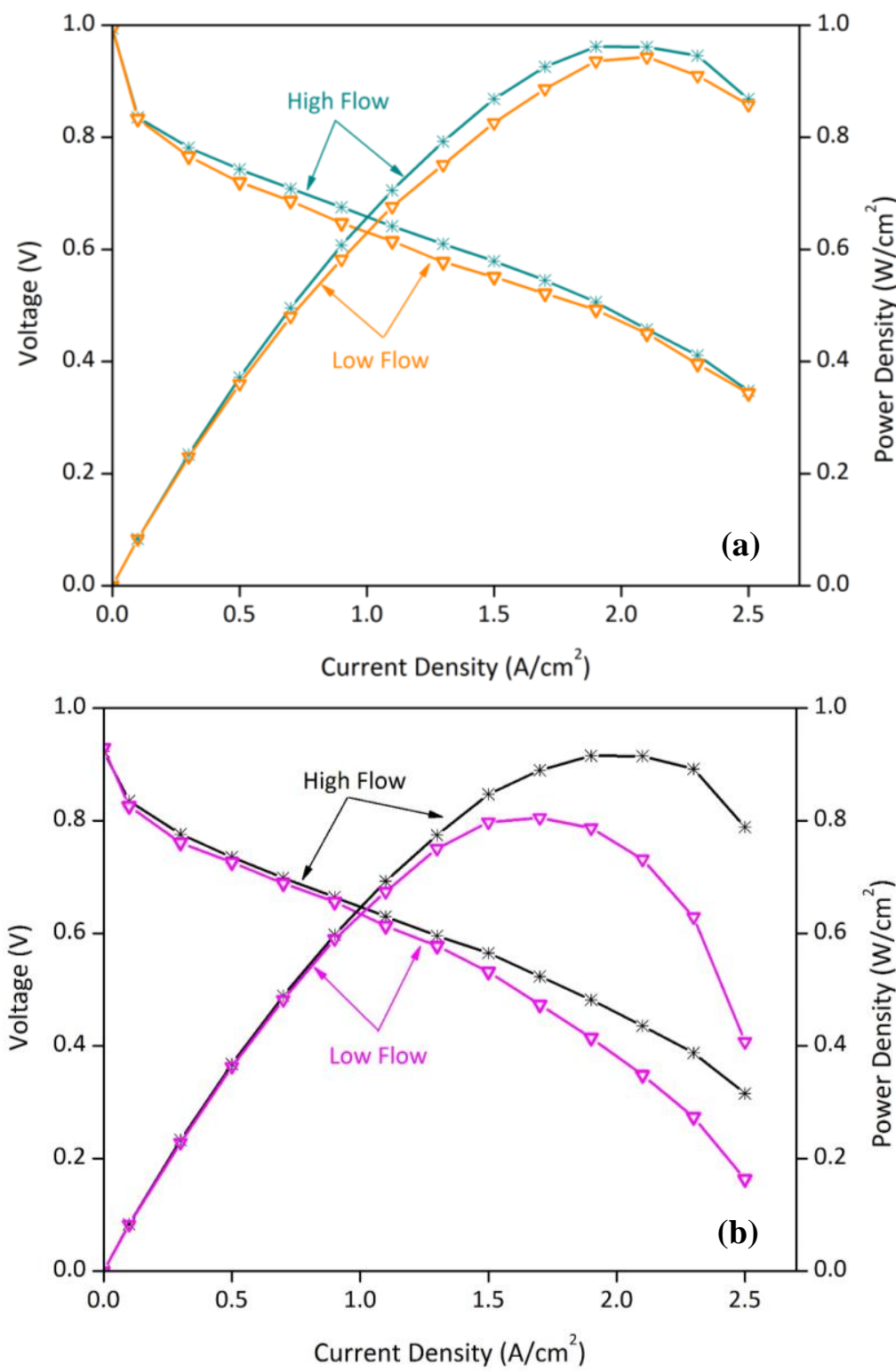

Figure 2. Polarization and power curves of the MEAs with the catalyst layers (CLs) and membranes made of either (a) SSC or (b) LSC ionomers under low and high flow conditions.

maintained, regardless of the flow conditions. By comparing Figs. 2 (a) and 2 (b), it can be concluded that the MEA with the SSC ionomer-based CLs and membrane yields better cell performance under high- and low-flow conditions than its LSC ionomer-based counterpart; such a performance superiority may be attributed to the SSC-based components' higher stability, crystallinity, and proton-transport capability [11].

Cell temperature is another critical parameter affecting the capability of the constituents in dealing with mass, heat, and electron transport. Thus, performances of the MEAs with the CLs and membranes based on either SSC or LSC ionomers are investigated at two different cell temperatures, namely, at $75^{\circ} \mathrm{C}$ and $95^{\circ} \mathrm{C}$. These temperatures are specifically chosen, because the LSC-ionomers and membranes demonstrate the best performance at the cell temperature of $75^{\circ} \mathrm{C}$; meanwhile, it is also known that their effective operation is limited to $100^{\circ} \mathrm{C}$. Hence, temperatures chosen are expected to provide a better understanding of the differences between the SSC and 

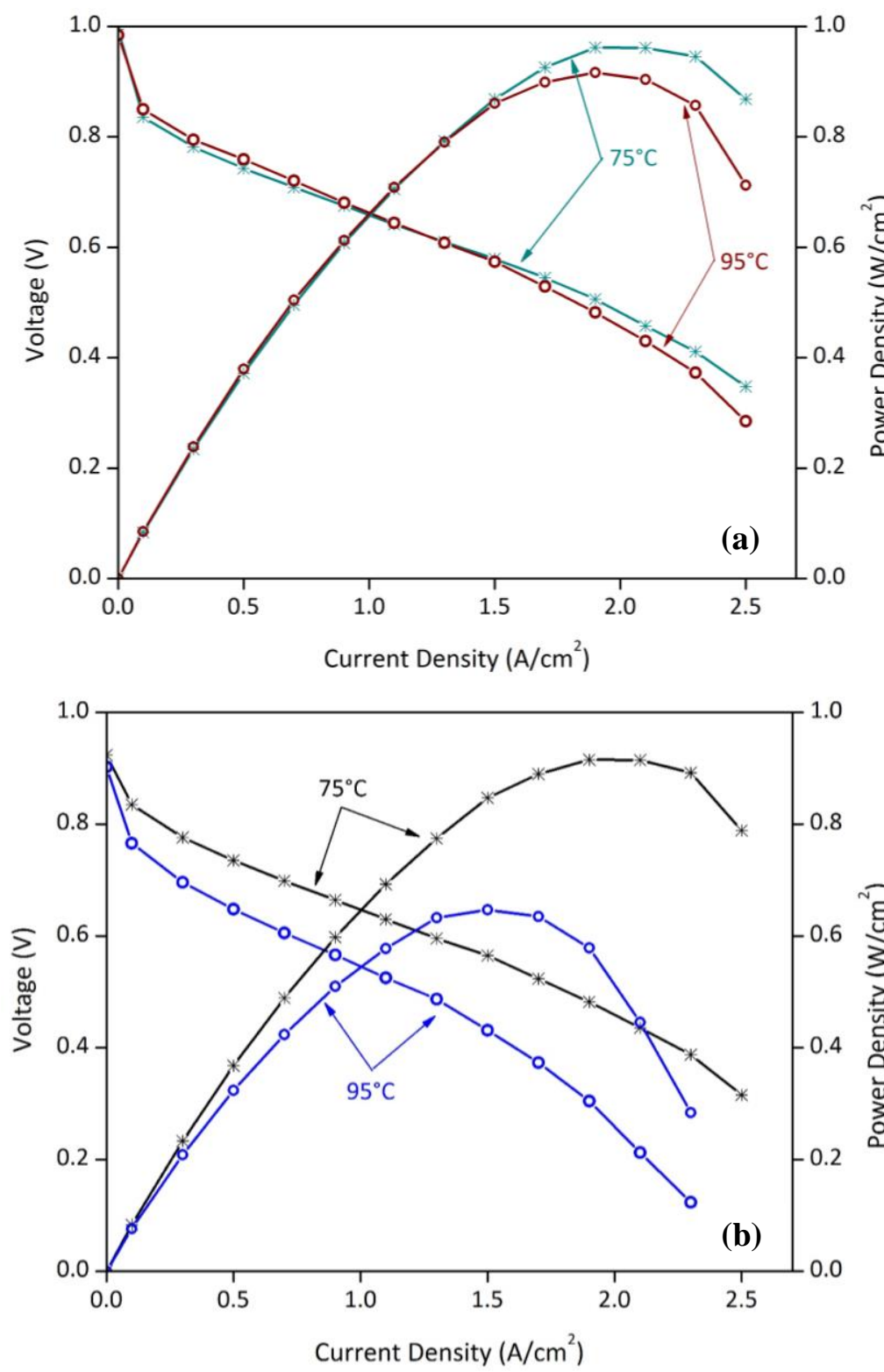

Figure 3. Polarization and power curves of the MEAs with the catalyst layers (CLs) and membranes made of either (a) SSC or (b) $\mathrm{LSC}$ ionomers at $75^{\circ}$ and $95^{\circ} \mathrm{C}$.

LSC ionomers and membranes. For a proper comparison, the other operating parameters are held constant during performance testing, such that the flow rates, back pressures, and RHs of the air and hydrogen streams are set to 4.45 and $9.00 \mathrm{l} / \mathrm{min}, 35 \mathrm{kPag}$, and $100 \%$, respectively. As seen from Fig. 3, the MEA with the SSC ionomer-based CLs and membrane shows virtually the same performance in activation-polarization and ohmic-polarization dominant regions, as the operating temperature is increased from $75^{\circ} \mathrm{C}$ to $95^{\circ} \mathrm{C}$, while a slight drop in cell voltage is detected at high current densities. For example, at the constant current density of $2.50 \mathrm{~A} / \mathrm{cm}^{2}$, the performance of the MEA with the SSCbased ionomer and membrane drops almost $17 \%$, as the cell temperature is increased from $75^{\circ} \mathrm{C}$ to $95^{\circ} \mathrm{C}$. However, the MEA with the LSC ionomer-based CLs and membrane performs quite differently in activation-, ohmic-, and concentration-polarization dominant regions with increasing cell temperature (see Fig. 3(b)). The drop in cell voltage is even more substantial at high current densities, such that at the current density of $2.30 \mathrm{~A} / \mathrm{cm}^{2}$, a voltage drop of about $68 \%$ is detected. The performance differences between the two MEAs may be better understood by taking a closer look at the maximum power densities obtained at the cell temperatures of $75^{\circ} \mathrm{C}$ and $95^{\circ} \mathrm{C}$. The MEA with the SSC ionomer-based CLs and membrane yields the maximum power densities of 0.96 $\mathrm{W} / \mathrm{cm}^{2}$ and $0.92 \mathrm{~W} / \mathrm{cm}^{2}$ at the cell temperatures of $75^{\circ} \mathrm{C}$ and $95^{\circ} \mathrm{C}$, respectively. However, the maximum power densities obtained at the cell temperatures of $75^{\circ} \mathrm{C}$ and $95^{\circ} \mathrm{C}$ from the MEA with the LSC ionomer-based CLs and membrane are $0.92 \mathrm{~W} / \mathrm{cm}^{2}$ and $0.65 \mathrm{~W} / \mathrm{cm}^{2}$, respectively. Clearly, increasing cell temperature results in severe deterioration in performance of the MEA with the LSC ionomer-based CLs and membrane, but only slight deterioration in performance of the MEA with the SSC ionomer-based CLs and membrane - a finding indicating that the SSC ionomer-based cell components are far less sensitive to changes in the operating temperature; or alternatively, the proton transport in the SSC ionomer and membrane is effectively maintained at elevated temperatures. This behaviour could be related to the high crystallinity and better proton transport capability of the SSC ionomer at elevated temperatures. Further, owing to their higher crystallinity and lower equivalent weight, the SSC ionomer and membrane experience less swelling, which is not only useful for preserving the interfacial characteristics at the threephase boundary but also for maintaining the electrode microstructural characteristics over a wide range of cell temperature. Furthermore, due to their lower equivalent weight, the SSC ionomer and membrane probably retain adequate water, which in turn enables them not to suffer from membrane dehydration, hence function well even at elevated temperatures. In the case of the MEA with the LSC ionomerbased CLs and membrane, high-temperature operation probably leads to degradation in the LSC-ionomer based components, i.e., excessive swelling, and thus both the interfacial and microstructural characteristics may not be maintained at high cell temperatures. The joint effect of these changes in turn leads to significant deterioration in the cell performance in both ohmic- and concentration-polarization dominant regions (see Fig. 3).

The effect of the RHs of the air and hydrogen streams on performances of the MEAs with the CLs and membranes made of either SSC or LSC ionomers are also investigated. Fig. 4 shows the power and polarization curves of the MEAs under the RHs of $55 \%$ and $100 \%$. Clearly, a decrease in the RHs of the air and hydrogen streams negatively impacts performances of both the MEAs. However, the MEA with the SSC ionomer-based CLs and membrane seems to experience less performance drop with decreasing RHs, compared to the one with the LSC ionomer-based CLs and membrane. For example, the MEA with the SSC ionomer-based CLs and membrane experiences a cell voltage drop of $13 \%$ at the constant current density of $1.50 \mathrm{~A} / \mathrm{cm}^{2}$, as the RHs are decreased from $100 \%$ to $55 \%$, while drop in cell voltage of the MEA with the LSC-based CLs and membrane is almost $46 \%$. The maximum power densities obtained for the SSC- and LSC-based MEAs at $55 \% \mathrm{RH}$ are $0.76 \mathrm{~W} / \mathrm{cm}^{2}$ and 0.55 $\mathrm{W} / \mathrm{cm}^{2}$, respectively. A noticeably better cell performance of the SSC-based MEA is probably due to the higher waterretention capability of the SSC ionomer and membrane, 

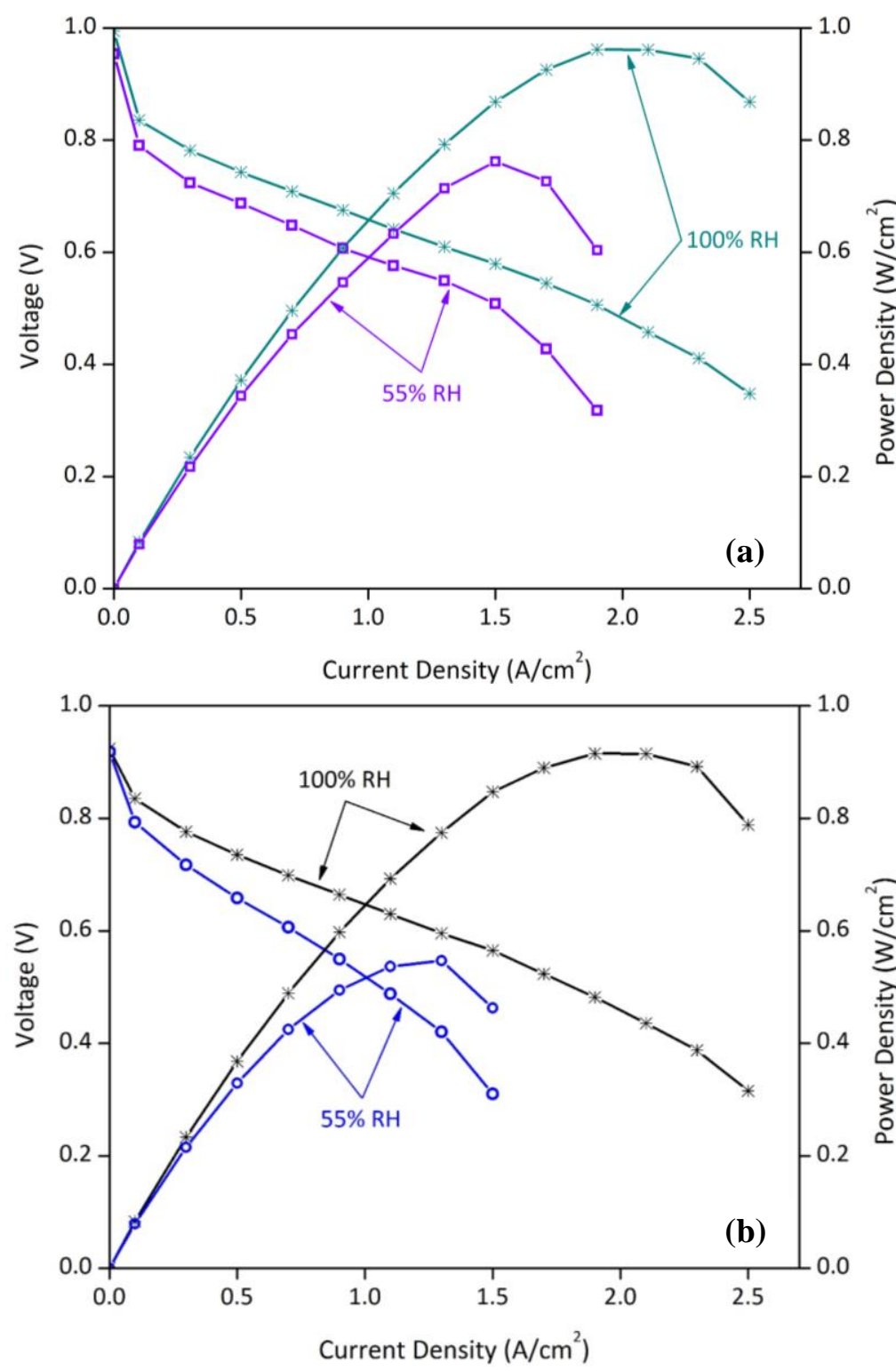

Figure 4. Power and polarization curves of the MEAs with the catalyst layers (CLs) and membranes made of either (a) SSC or (b) LSC ionomer under different RHs of air and hydrogen streams.

compared to their LSC counterparts, since being able to operate well under low-humidity conditions requires effective proton-transport capability. This advanced proton-transport capability is probably due to its lower equivalent weight than the LSC ionomer, which provides relatively higher number of hydrophilic sulfonic acid groups. The presence of more sulfonic acid groups inside both the ionomer and membrane yields effective proton transport even when the cell is operated under low RHs, thus the cell suffers less from ohmic polarization and performs much better.

\section{CONCLUSIONS}

In this study, the effect of ionomer structure in the membrane and catalyst layer (CL) on the performance of proton exchange membrane (PEM) fuel cells is experimentally investigated in a scaled-up cell $\left(45 \mathrm{~cm}^{2}\right)$. Short-side-chain (SSC) ionomer and membrane are applied in a membrane-electrode assembly
(MEA), and cell performance of this MEA is investigated over a wide range of operating conditions: different cell temperatures $\left(75^{\circ} \mathrm{C}\right.$ and $\left.95^{\circ} \mathrm{C}\right)$, air and hydrogen flow rates (low- and high-flow conditions), and air and hydrogen relative humidities (100\% RH and 55\% RH). Comparison is made with an MEA based on long-side-chain (LSC) ionomer-based CLs and membrane. The morphological and microstructural characteristics of the SSC ionomer-based CLs are investigated, and comparison is made with the LSC ionomer-based CLs. The pore characteristics of the electrodes with these CLs are also investigated. Results indicate that the SSC ionomer not only offers more uniform surface morphology and microstructure, but also provides desirable coverage characteristics; adequate Pt-C coverage without blocking the pores. Thus, the SSC ionomer-based electrode has a relatively higher porosity $(73.10 \%)$ than the LSC ionomer-based electrode $(68.80 \%)$. The performance test indicates that the MEA with the SSC ionomer-based CLs and membrane shows superior performance than the one with the LSC ionomerbased CLs and membrane under all the studied conditions. In particular, the SSC ionomer and membrane are found to be quite suitable for high-temperature operation, due to their favorable water-retention capability (hence proton-transport capability) at high temperatures. Lastly, the MEA based on SSC-ionomer and membrane is found to be less sensitive to variations in operating conditions.

\section{REFERENCES}

[1] Ozden A, Alaefour IE, Shahgaldi S, Li X, Colpan CO, Hamdullahpur F, Chapter 2.28 - Gas Diffusion Layers for PEM Fuel Cells: Ex- and In-Situ Characterization. Exergetic, Energetic and Environmental Dimensions. doi:10. 1016/B97.-0-12813734-5. 0004.-8. 1

[2] Shahgaldi S, Hamelin J. Stability study of ultra-low Pt thin film on TiO2-C coreshell structure and TiO2encapsulated in carbon nanospheres as cathode catalyst in PEMFC. Fuel 2015;150:645-55. doi:10.1016/j.fuel.2015.02.002.

[3] Ozden A, Shahgaldi S, Zhao J, Li X, Hamdullahpur F. Assessment of graphene as an alternative microporous layer material for proton exchange membrane fuel cells. Fuel 2018;215:726-34. doi:10.1016/j.fuel.2017.11.109.

[4] Ercelik M, Ozden A, Seker E, Colpan CO. Characterization and performance evaluation of $\mathrm{PtRu} / \mathrm{CTiO} 2$ anode electrocatalyst for DMFC applications. Int $\mathbf{J}$ Hydrogen Energy 2016;2:1-12. doi:10.1016/j.ijhydene.2016.12.020.

[5] Bose S, Kuila T, Nguyen TXH, Kim NH, Lau KT, Lee JH. Polymer membranes for high temperature proton exchange membrane fuel cell: Recent advances and challenges. Prog Polym Sci 2011;36:813-43. doi:10.1016/j.progpolymsci.2011.01.003.

[6] Shahgaldi S, Hamelin J. Improved carbon nanostructures as a novel catalys support in the cathode side of PEMFC: A critical review. Carbon N Y 2015;94:705-28. doi:10.1016/j.carbon.2015.07.055.

[7] Ozden A, Ercelik M, Ozdemir Y, Devrim Y, Colpan CO. Enhancement of direct methanol fuel cell performance through the inclusion of zirconium phosphate. Int $\mathrm{J}$ Hydrogen Energy 2017;42:21501-17. doi:10.1016/j.ijhydene.2017.01.188.

[8] Ozden A, Ercelik M, Devrim Y, Colpan CO, Hamdullahpur F. Evaluation of sulfonated polysulfone/zirconium hydrogen phosphate composite membranes for direct methanol fuel cells. Electrochim Acta 2017;256:196-210. doi:10.1016/j.electacta.2017.10.002.

[9] Ercelik M, Ozden A, Devrim Y, Colpan CO. Investigation of Nafion based composite membranes on the performance of DMFCs. Int J Hydrogen Energy 2016;42:2658-68. doi:10.1016/j.ijhydene.2016.06.215

[10] Shahgaldi S, Alaefour IE LX. Influence of Ionomer Structures and Ratios on Performance and Degradation of PEM Fuel Cells 2018;83:71-8.

[11] Lei C, Bessarabov D, Ye S, Xie Z, Holdcroft S, Navessin T. Low equivalent weight short-side-chain perfluorosulfonic acid ionomers in fuel cell cathode catalyst layers. J Power Sources 2011;196:6168-76. doi:10.1016/j.jpowsour.2011.03.024 